ARTICLE

\title{
Characterization of the WENDI-II REM Counter for its Application at MedAustron
}

\author{
Lukas JÄGERHOFER ${ }^{1, *}$, Eduard FELDBAUMER ${ }^{1}$, Doris FORKEL-WIRTH ${ }^{1}$, Chris THEIS ${ }^{1}$, Helmut VINCKE ${ }^{1}$, \\ Yosuke IWAMOTO $^{5}$, Masayuki HAGIWARA ${ }^{2}$, Daiki SATOH ${ }^{5}$, Hiroshi IWASE ${ }^{2}$, Hiroshi YASHIMA ${ }^{6}$, \\ Tetsuo MATSUMOTO ${ }^{7}$, Akihiko MASUDA $^{7}$, Jun NISHIYAMA ${ }^{7}$, Takashi NAKAMURA ${ }^{4}$, Tatsuhiko SATO ${ }^{5}$, \\ Yoshihiro NAKANE ${ }^{5}$, Hiroshi NAKASHIMA ${ }^{5}$, Yukio SAKAMOTO ${ }^{5}$, Atsushi TAMII ${ }^{3}$ and Kichiji HATANAKA ${ }^{3}$ \\ ${ }^{1}$ CERN, 1211 Geneve 23, Switzerland \\ ${ }^{2}$ KEK, 1-1 Oho, Tsukuba, Ibaraki 305-0801 Japan \\ ${ }^{3}$ RCNP, 10-1 Mihogaoka, Ibaraki, Osaka, 567-0047, Japan \\ ${ }^{4}$ CYRIC Tohoku University, 6-3 Aoba, Aramaki, Aoba-ku, Sendai, Miyagi, 980-8578, Japan \\ ${ }^{5}$ JAEA Japan Atomic Energy Agency, 4-49 Muramatsu, Tokai-mura, Naka-gun, Ibaraki 319-1184, Japan \\ ${ }^{6}$ Kyoto University, Kyoto 606-8502, Japan \\ ${ }^{7}$ Advanced Industrial Science and Technology, 1-3-1 Kasumigaseki, Chiyoda-ku, Tokyo 100-8921 Japan
}

\begin{abstract}
MedAustron is an accelerator facility for proton-, ion-therapy and research which is going to be built in Austria. The facility uses proton beams for scientific applications with kinetic energies up to $800 \mathrm{MeV}$ and therefore, neutron dominated fields up to several hundred $\mathrm{MeV}$ can be expected behind shielding. During the operation of the accelerator dose levels have to be monitored with appropriate equipment, such as the WENDI-II REM counter ${ }^{\mathrm{a}}$, to prove that the dose does not exceed the legal limits inside accessible areas. In order to demonstrate the appropriateness of the WENDI II, measurements at the Research Center for Nuclear Physics (RCNP) facility at Osaka University of Japan were conducted in quasi monoenergetic neutron beams of $246 \mathrm{MeV}$ and $389 \mathrm{MeV}$. In the literature the WENDI-II detector was already characterized in mixed fields at CERN as well as quasi monoenergetic fields up to $173 \mathrm{MeV}$ at the T. Svedberg Laboratory. This paper provides a characterization for neutron fields at higher energies and compares the measurements with FLUKA simulations. Additionally, a series of measurements with iron shielding of different dimensions was conducted as the WENDI-II will be utilized behind thick shielding at MedAustron.
\end{abstract}

KEYWORDS: shielding, REM counter, neutrons, field calibration, FLUKA, benchmark

\section{Introduction}

MedAustron is an Austrian project funded by various public stakeholders in order to design and build a proton- as well as ion-therapy facility which will also include a research branch. For the latter proton beams up to $800 \mathrm{MeV}$ will be used while proton- and carbon ion beams with a maximum energy of $250 \mathrm{MeV}$ and $400 \mathrm{MeV} /$ nucleon respectively will be utilized for cancer treatment. During the operation of the accelerator appropriate detectors have to be used to monitor the compliance of dose rates in accessible areas with legal limits. Since the spectral composition of radiation fields behind iron and concrete shielding will comprise neutrons with a wide range of energies, an adequate monitor like the WENDI-II has to be chosen. A series of measurements was performed in quasi monoenergetic neutron fields at the $\mathrm{RCNP}^{1)}$ facility in Osaka, Japan, for demonstrating that the WENDI-II is an appropriate device to monitor ambient dose equivalent rates in high-energy neutron dominated fields. This included measurements with different dimensions of iron shielding and measurements without shielding. Detailed Monte-Carlo-Simulations with

*Corresponding author, E-mail:Lukas.Jaegerhofer@cern.ch

(c) 2011 Atomic Energy Society of Japan, All Rights Reserved.

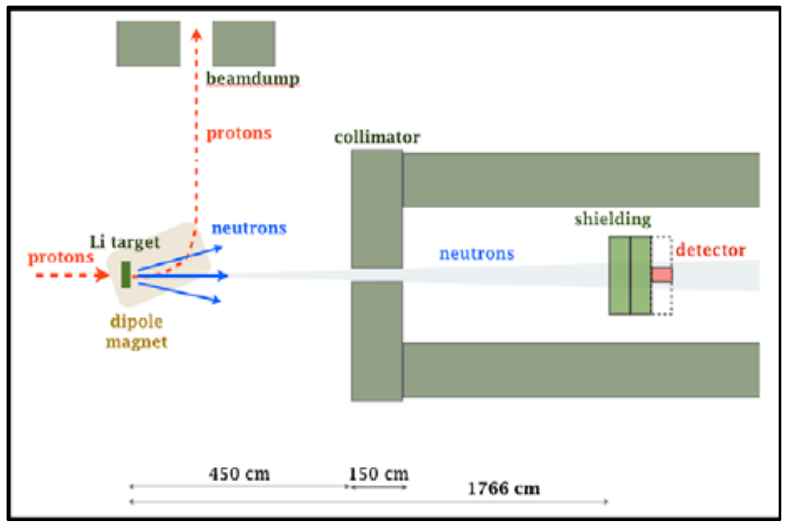

Fig. 1 Experimental layout at the RCNP facility ${ }^{4)}$

FLUKA $^{2,3)}$ were performed and compared to the measurements.

\section{Experimental Methods}

\section{Facility}

All measurements were performed with a WENDI-II REM meter at the RCNP in Osaka. This facility provides

\footnotetext{
${ }^{\mathrm{a}}$ Thermo Electron Corporation, Frauenauracher Strasse 96, 91056 Erlangen, Germany
} 


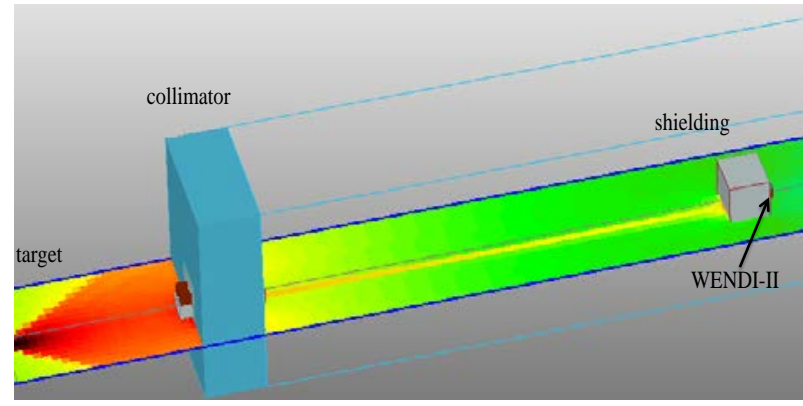

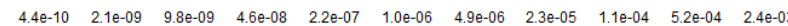

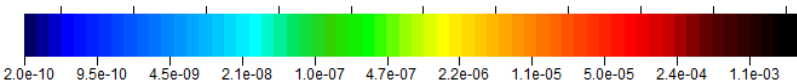

Fig. 2 Visualisation of the FLUKA geometry including the simulated neutron fluence in $1 / \mathrm{cm}^{2} /$ primary-neutron. The walls and the floor of tunnel and target area have been omitted in this picture for better understanding. ${ }^{5)}$ The source neutron spectra cut off of is $2.5 \mathrm{MeV}$.

quasi monoenergetic neutron beams using the ${ }^{7} \mathrm{Li}(\mathrm{p}, \mathrm{n}){ }^{7} \mathrm{Be}$ reaction in a $10 \mathrm{~mm}$ thick target. Neutrons with peak energies of $246 \mathrm{MeV}$ and $389 \mathrm{MeV}$ were collimated into a concrete tunnel to irradiate the WENDI-II detector. A Faraday cup measures the primary proton beam current at the target. Charged particles, emitted together with neutrons from the target are bent into a beam dump. The $\gamma$-radiation component, produced in the target, does not contribute to the measurement since signals originating from photons are rejected by the WENDI-II REM meter. Figure 1 shows the layout of the target area and the tunnel at the RCNP facility. As can be seen various configurations of iron shielding were placed in front of the detector inside the tunnel.

\section{Detector}

The WENDI-II REM meter was equipped with an FH $40 \mathrm{G}^{\mathrm{a}}$ survey meter by the Thermo Electron Corporation which every second sent the dose measurement in $\mu \mathrm{Sv} / \mathrm{h}$ to a computer via a serial connection. During the subsequent data analysis the value was normalized with the actual proton current at the target. It should be noted that the WENDI-II REM meter is intrinsically calibrated with a ${ }^{252} \mathrm{Cf}$ source to measure $H^{*}(10)$.

\section{Simulation Methods}

For the simulation of the neutron fields the Monte Carlo Code FLUKA was used. A detailed geometry of the RCNP layout, including the target area, the collimator, the tunnel and the shielding was included in the FLUKA input. The geometry was built with the solid modeler SimpleGeo. ${ }^{5)}$ Figure 2 shows a rendering of the geometry with $100 \mathrm{~cm}$ of iron shielding including the simulated neutron fluence distribution. During the measurements Iwamoto et al. ${ }^{6}$ recorded neutron spectra originating from the Li-target in seven different angles up to $35^{\circ}$. The spectra were measured down to an energy of $2.5 \mathrm{MeV}$. In order to model the source in the FLUKA simulation as accurately as possible a routine was developed which sampled the neutron spectra under the associated angle. The resulting neutron fluence was determined in a volume corresponding to the size and the VOL. 2, OCTOBER 2011
Table 1 Results of FLUKA simulations compared to the WENDI-II measurements at RCNP. The FLUKA dose rates are based on the full spectral field composition at RCNP whereas the WENDI-II results are based on its intrinsic ${ }^{252} \mathrm{Cf}$ calibration.

\begin{tabular}{rccccc}
\hline Shielding & \multicolumn{2}{c}{ FLUKA } & Ratio & \multicolumn{2}{c}{ WENDI-II } \\
\hline 246 MeV: & $\left(\frac{\mu S v}{h \mu A}\right)$ & $(\%)$ & & $\left(\frac{\mu S v}{h \mu A}\right)$ & $(\%)$ \\
no shielding: & 2955 & \pm 18 & $\mathbf{0 . 5 8}$ & 5099 & \pm 11 \\
40 cm iron: & 4692 & \pm 19 & $\mathbf{0 . 9 3}$ & 5046 & \pm 12 \\
70 cm iron: & 1370 & \pm 18 & $\mathbf{0 . 8 9}$ & 1532 & \pm 11 \\
100 cm iron: & 402 & \pm 19 & $\mathbf{0 . 9 1}$ & 442 & \pm 12 \\
389 MeV: & & & & & \\
no shielding: & 3407 & \pm 18 & $\mathbf{0 . 4 4}$ & 7695 & \pm 11 \\
40 cm iron: & 6107 & \pm 18 & $\mathbf{0 . 8 2}$ & 7488 & \pm 11 \\
70 cm iron: & 2283 & \pm 18 & $\mathbf{0 . 8 5}$ & 2679 & \pm 11 \\
100 cm iron: & 735 & \pm 18 & $\mathbf{0 . 9 4}$ & 780 & \pm 10 \\
\hline
\end{tabular}

position of the detector. At the same time the ambient dose equivalent was obtained by direct convolution with fluence to dose conversion functions implemented in FLUKA.")

\section{Results and Discussion}

Table 1 shows the results of the FLUKA simulations compared to the associated measurements at the RCNP facility. All results have been normalized to $1 \mu \mathrm{A}$ of proton current sent to the target. The column in the middle indicates the ratio between simulation and measurement. All given uncertainties include statistical and systematic errors, of which the main contributions arise from uncertainties in the sampled spectra $(\sim 15 \%)$ and the uncertainties in the measurement of the primary proton beam at the target $(\sim 10 \%) .{ }^{6}$ )

While the FLUKA results shown in Table 1 are based on the full field composition, it should be noted that the WENDI-II results include the detector's intrinsic calibration in a ${ }^{252} \mathrm{Cf}$ field. As a consequence of this intrinsic calibration factor each count $\left({ }^{3} \mathrm{He}(\mathrm{n}, \mathrm{p})^{3} \mathrm{H}\right.$ reaction) triggered by the RCNP neutron radiation field is converted into a dose value based on the response to a typical ${ }^{252} \mathrm{Cf}$ neutron spectrum.

According to Fig. 3, the non-attenuated ("no-shielding") neutron spectrum at RCNP is dominated by much higher energies than those encountered for a Californium source. This circumstance was suspected to account for the observed deviation between the measured and the simulated dose and triggered a more detailed analysis. In general, neutron spectra behind thick shielding are found to be softer. Thus, it can be understood that the ${ }^{252} \mathrm{Cf}$ based calibration is more suitable for these cases which explains the better agreement of measurements behind shielding.

A possible way to account for the effect of different radiation fields is obtained by introducing specific correction factors. These correction factors are determined by recording neutron spectra at the measurement location for each shielding configuration which are then convoluted with the pure 


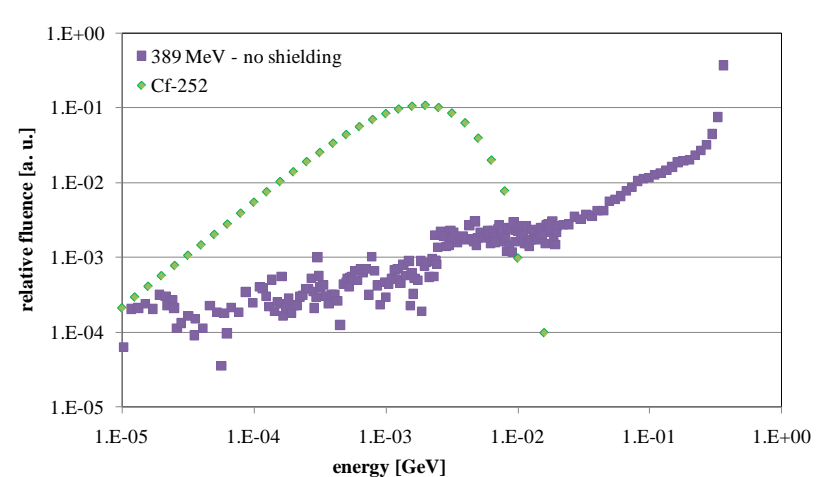

Fig. 3 Neutron fluence spectrum at RCNP, compared to the characteristic peak of the neutron fluence spectrum of a ${ }^{252} \mathrm{Cf}$ source. Neutrons below $10 \mathrm{keV}$ are not shown, since their contribution to the dose is negligibly small for the "no shielding" case. $^{8)}$

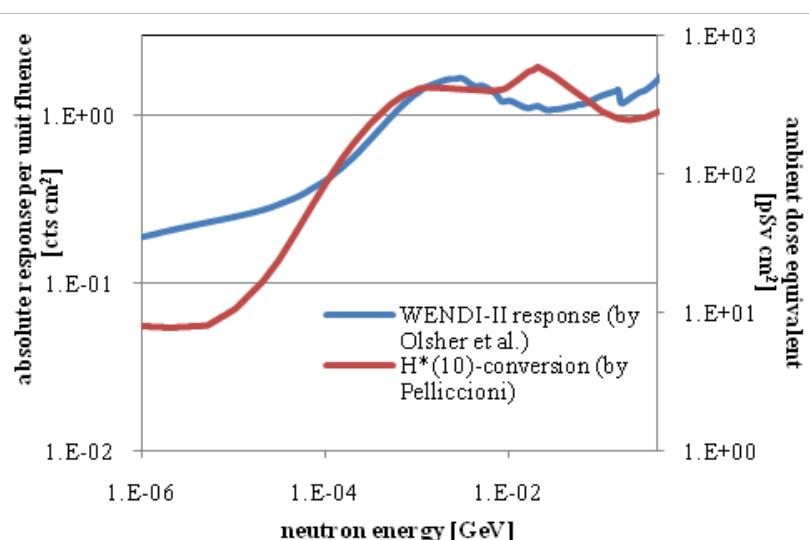

Fig. 4 Pure response function of the WENDI-II, calculated by Olsher et al. with $\mathrm{MCNPX}^{9)}$ in relation to the ambient dose equivalent conversion function. For a detailed discussion of the comparison please refer to Gutermuth et al. ${ }^{10)}$

WENDI-II response function (see Fig. 4) $)^{9)}$ as well as with the conversion function for ambient dose equivalent from Pelliccioni. ${ }^{7)}$ The ratio of the two integrated energy spectra eventually yields field calibration factors in units of counts $/ \mu \mathrm{Sv}$. Subsequently these results were divided by the sensitivity of the detector (counts/ $/ \mathrm{Sv}_{\mathrm{Cf}-252}$ ) given by the manufacturer to determine the correction factors. Figure 4 shows the pure WENDI-II response function without any corrections as calculated by Olsher et al. ${ }^{9)}$ using the Monte Carlo code MCNPX in comparison to the conversion function for ambient dose equivalent according to Pelliccioni. ${ }^{7}$

As can be seen in Fig. 4 the response function in general reflects the ambient dose equivalent conversion function fairly well. Even though the response overestimates neutrons in the energy range below $0.1 \mathrm{MeV}$ and underestimates in the range of $\sim 6 \mathrm{MeV}$ to $\sim 60 \mathrm{MeV}$. As also shown in Fig. 4 one can expect some overestimation of $H^{*}(10)$ for neutron fields clearly dominated by energies above $100 \mathrm{MeV}$ like those encountered at RCNP without shielding (Fig. 3). This explains the overestimation of the WENDI-II results in Table 1 for the "no shielding" cases.

Figure 5 illustrates a plot of the determined correction factors which were then used to adapt the WENDI-II results to the real field composition at RCNP. For the correction

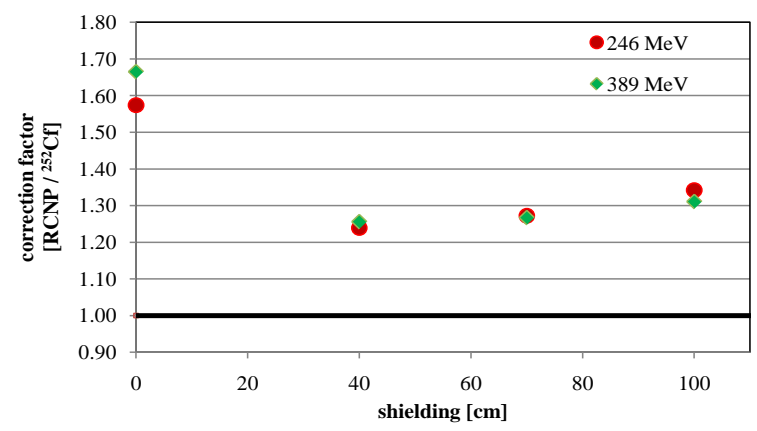

Fig. 5 Correction factors for different shielding dimensions

Table 2 Results of FLUKA simulations compared to WENDI-II measurements after field calibration

\begin{tabular}{rccccc}
\hline Shielding & FLUKA & Ratio & $\begin{array}{c}\text { WENDI-II } \\
\text { (field calibrated } \\
\text { representation) }\end{array}$ \\
\hline 246 MeV: & $\left(\frac{\mu S v}{h \mu A}\right)$ & $(\%)$ & & $\left(\frac{\mu S v}{h \mu A}\right)$ & $(\%)$ \\
no shielding: & 2955 & \pm 18 & $\mathbf{0 . 9 1}$ & 3239 & \pm 24 \\
40 cm iron: & 4692 & \pm 19 & $\mathbf{1 . 1 5}$ & 4073 & \pm 24 \\
70 cm iron: & 1370 & \pm 18 & $\mathbf{1 . 1 4}$ & 1205 & \pm 24 \\
100 cm iron: & 402 & \pm 19 & $\mathbf{1 . 2 2}$ & 330 & \pm 24 \\
$\mathbf{3 8 9}$ MeV: & & & & & \\
no shielding: & 3407 & \pm 18 & $\mathbf{0 . 7 4}$ & 4620 & \pm 24 \\
40 cm iron: & 6107 & \pm 18 & $\mathbf{1 . 0 2}$ & 5962 & \pm 24 \\
70 cm iron: & 2283 & \pm 18 & $\mathbf{1 . 0 8}$ & 2114 & \pm 24 \\
100 cm iron: & 735 & \pm 18 & $\mathbf{1 . 2 3}$ & 595 & \pm 24 \\
\hline
\end{tabular}

factors uncertainties of about $20 \%$ have to be taken into account, which arise from intrinsic uncertainties of the response function and the source spectra.

Table 2 shows a comparison between the simulation result and the corrected measurements. For energies above $100 \mathrm{MeV}$ uncertainties of up to $15 \%$ are introduced by the nuclear models, which form the basis of the correction factor calculation.

Figure 6 shows a graphical representation of the results in Table 2. It should be noted that the measurements without shielding were performed at RCNP at greater distance from the target since preparations for the shielding experiments had been taking place at the closer position. This explains the much lower dose rates in terms of absolute values and as a consequence the measurement results from this configuration are not included in Fig. $\mathbf{6}$ for reasons of clarity.

As can be seen from Table 2 the application of the field calibration obviously improved the agreement for the nonshielded cases which showed the biggest deviation of the impinging neutron spectrum with respect to a ${ }^{252} \mathrm{Cf}$ field. However, for neutron energies of $389 \mathrm{MeV}$ a tendency to better agreement can still be observed for the shielding measurements. This could be due to the fact that the nuclear models, on which the response function by Olsher et al. is based, have increasing uncertainties for higher energies. ${ }^{9}$

PROGRESS IN NUCLEAR SCIENCE AND TECHNOLOGY 


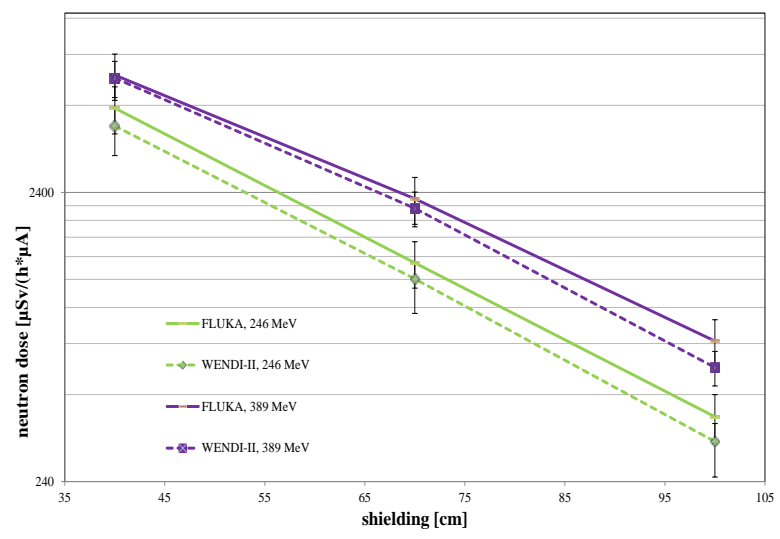

Fig. 6 Plot of the FLUKA results and the field calibrated WENDI-II measurements.

Figure 7 shows the cumulative contribution to the WENDI-II counts as a function of the neutron energy in two configurations: First, a configuration in which the detector is located behind $100 \mathrm{~cm}$ of iron; second, a configuration without shielding. The measurement readings without shielding are clearly dominated by high energy neutrons while those originating from a configuration with shielding show a significant contribution from neutrons of lower energy. Therefore, it can be understood that larger uncertainties at higher energies can be expected to have a more notable impact on the calculated field specific correction factor. This fact can explain the bigger deviation between simulation and measurement for the "non shielding" cases compared to the shielded cases in Table 2.

\section{Conclusion}

$\mathrm{H}^{*}(10)$ measurement results of the WENDI-II REM meter can be reproduced by FLUKA for energies of a few hundred $\mathrm{MeV}$ with satisfying agreement. In contrast to the simulation the WENDI-II measurements yield dose rates based on a standard ${ }^{252} \mathrm{Cf}$ calibration factor. If the actual field composition deviates notably from the energy range covered by such a source, it is prudent to determine field specific calibration factors for the WENDI-II. These values can be used to adapt the intrinsic source based count-to-dose relation of the monitor to the respective field composition. The calculation of these field specific calibration factors can be done, but requires some effort since a detailed geometry of the surrounding areas has to be built. Depending on the complexity of the configuration, time consuming simulations have to be performed. In a facility like MedAustron this could be done for neuralgic points where REM meters will be installed permanently. According to the results presented here, safety margins should be applied for mobile devices. Since the response function of the WENDI-II REM meter and the ambient dose conversion function show good agreement $^{10)}$ (see Fig. 4) and since the calculated correction factors are within 20 percent for various shielding configurations, the WENDI-II can be considered to be an appropriate device to measure $\mathrm{H}^{*}(10)$ neutron dose behind shielding for primary beam energies of $\sim 250 \mathrm{MeV}$ and $\sim 400 \mathrm{MeV}$ even

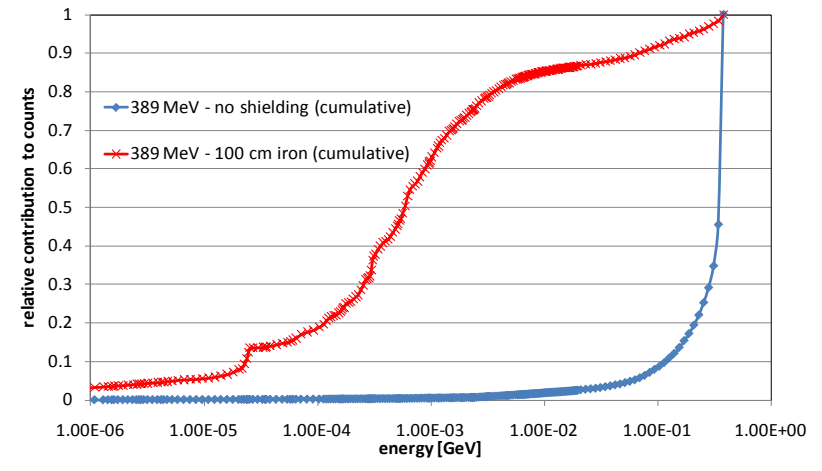

Fig. 7 Cumulative contribution to counts of the WENDI-II detector as a function of neutron energy for two different shielding configurations.

without a field specific calibration procedure. Yet, it is advisable to include the aforementioned uncertainty of $20 \%$.

Future work in this field will include the simulations of concrete shielding for comparison with measurements taken at RCNP. Furthermore, the development of a response function for the WENDI-II REM meter based on FLUKA calculations is envisaged in order to compare these results also to already published material obtained with MCNPX. ${ }^{9)}$

\section{Acknowledgements}

The authors would like to thank EBG MedAustron for the financial support and Richard Olsher for his patient and quick responses to the authors' e-mails. The authors would also like to thank Stefan Roesler and Hannes Bartosik for extensive reviewing.

\section{References}

1) S. Taniguchi, N. Nakao, T. Nakamura, H. Yashima, Y. Iwamoto, D. Satoh, Y. Nakane, H. Nakashima, T. Itoga, A. Tamii, K. Hatanaka, "Development of a quasi-monoenergetic neutron field using the ${ }^{7} \mathrm{Li}(\mathrm{p}, \mathrm{n})^{7} \mathrm{Be}$ reaction in the energy range from 250 to $390 \mathrm{MeV}$ at RCNP," Radiat. Prot. Dosim., 126[1-4], 23-27 (2007).

2) A. Fasso, A. Ferrari, J. Ranft, P. R. Sala, FLUKA: a multi particle transport code, CERN Yellow Report, INFN/TC_05/11, SLAC-R-773 (2005).

3) G. Battistoni, S. Muraro, P. R. Sala, F. Cerutti, A. Ferrari, S. Roesler, A. Fasso', J. Ranft, "The FLUKA code: Description and benchmarking," Proceedings of the Hadronic Shower Simulation Workshop 2006, Fermilab 6--8 September 2006, M. Albrow, R. Raja (eds.), AIP Conference Proceeding 896, 31-49, (2007).

4) H. Iwase, M. Hagiwara, Y. Iwamoto, D. Satoh, H. Yashima, T. Matsumoto, J. Nishiyama, A. Masuda, H. Harano, T. Sato, Y. Nakane, T. Itoga, C. Theis, E. Feldbaumer, L. Jaegerhofer, C. Pioch, V. Mares, Y. Sakamoto, H. Nakashima, A. Tamii, T. Nakamura, "Benchmark experiment of neutron penetration through iron and concrete shields using 246 and $387 \mathrm{MeV}$ quasi-monoenergetic neutrons part-I: Measurement and calculation of neutron depth-dose distribution," to be published in SATIF-10 proceedings (2010).

5) C. Theis, K. H. Buchegger, M. Brugger, D. Forkel-Wirth, S. Roesler, H. Vincke, "Interactive three dimensional visualization and creation of geometries for Monte Carlo calculations," Nucl. Instr. Meth. Phys. Res., A562, 827-829 (2006).

6) Y. Iwamoto, M. Hagiwara, D. Satoh, H. Iwase, H. Yashima, T. Itoga, T. Sato, Y. Nakane, H. Nakashima, Y. Sakamoto, T. 
Matsumoto, A. Masuda, J. Nishiyama, A. Tamii, K. Hatanaka, C. Theis, E. Feldbaumer, L. Jaegerhofer, C. Pioch, V. Mares, T. Nakamura, "Characterization of quasi-monoenergetic neutron energy spectra using ${ }^{7} \mathrm{Li}(\mathrm{p}, \mathrm{n})$ reactions at 246 $389 \mathrm{MeV}$," to be published in SATIF-10 proceedings (2010).

7) M. Pelliccioni, "Overview of fluence-to-effective dose and fluence-to-ambient dose equivalent conversion coefficients for high energy radiation calculated using the FLUKA code," Radiat. Prot. Dosim., 88, 279-297 (2000).

8) International Organization for Standardization (ISO), Neutron reference radiations for calibrating neutron-measuring devic- es used for radiation protection purposes and for determining their response as a function of neutron energy, ISO 8529: 1989 (E) (1989).

9) R. H. Olsher, H. Hsu, A. Beverding, J. H. Kleck, W. H. Casson, D. G. Vasilik, R. T. Devine, "WENDI: An improved neutron REM meter,” Health Phys., 79[ 2], 170-180 (2000).

10) F. Gutermuth, T. Radon, G. Fehrenbacher, R. Siekmann, Test of the REM-Counter WENDI-II from Eberline in different energy-dispersed neutron fields, GSI Preprint 2004-05 (2004). 